Classification

Physics Abstracts

$61.30 \mathrm{E}-64.70 \mathrm{M}$

\title{
Between cylinders and bilayers : structures of intermediate mesophases of the SDS/water system
}

\author{
P. Kékicheff $(*)$ and B. Cabane $\left({ }^{+}\right)$ \\ LURE, Laboratoire CNRS-CEA-MEN, Bât. 209 D, Université Paris Sud-Orsay, 91405 Orsay, France
}

(Reçu le 3 février 1987, accepté le 22 mai 1987)

\begin{abstract}
Résumé. - L'empilement des molécules amphiphiles dans les phases ordonnées des systèmes lyotropes est décrit de manière classique dans deux cas extrêmes : la phase lamellaire où des bicouches de molécules amphiphiles sont séparées par des lamelles d'eau et la phase hexagonale, où les cylindres d'amphiphiles sont situés aux nœuds d'un réseau bidimensionnel hexagonal. Ces phases n'existent que pour certaines compositions du mélange eau/amphiphile ; entre ces extrêmes, s'étend une vaste plage de concentrations qui permet au système de passer d'une courbure nulle (bicouches) à une courbure finie (cylindres). Dans ces régions intermédiaires, on trouve un grand nombre de mésophases, chacune étant réduite à n'exister que sur une bande très étroite de concentrations $(\sim 1 \%)$. Afin de résoudre ce problème, nous avons délibérément créé un gradient spatial en concentration du mélange eau/amphiphile pour chaque échantillon; de tels échantillons sont déplacés devant le faisceau de rayons X de D24 au LURE, ce qui permet l'enregistrement de spectres de diffraction tous les $\sim 0,05 \%$. Pour le système SDS/eau nous avons identifié 4 mésophases intermédiaires: une phase tétragonale, une phase cubique, une phase rhomboédrique et une phase monoclinique bidimensionnelle. Ces structures montrent que le système peut passer d'une géométrie planaire à une géométrie cylindrique de manière quasiment continue.
\end{abstract}

\begin{abstract}
The classical geometries for the packing of amphiphile molecules in the ordered phases of lyotropic systems are the lamellar phase where bilayers of amphiphilic molecules are separated by water layers, and the hexagonal phase where cylinders of amphiphile are arranged on a two-dimensional hexagonal lattice. These phases only exist for definite compositions of the water/amphiphile system ; in between them, there usually lies a vast concentration region which allows the system to cross from zero curvature (bilayers) to finite curvature (cylinders). The problem with such intermediate regions is that they may contain a large number of mesophases, each limited to a very narrow range of compositions $(\sim 1 \%)$. To solve this problem we deliberately create a spatial gradient in the concentration of water/amphiphile of each sample ; such samples are scanned by the X-ray beam of a synchrotron source, allowing diffraction patterns to be recorded at concentration intervals $\sim 0.05 \%$. For the system SDS/water we have identified 4 intermediate mesophases : a tetragonal phase, a cubic phase, a rhomboedral phase and a two-dimensional monoclinic phase. These structures show that the system manages to cross from a planar geometry to a cylindrical geometry in a nearly continuous way.
\end{abstract}

\section{Introduction.}

Amphiphilic molecules made of polar heads attached to apolar tails tend to form condensed phases where polar/apolar contacts are avoided. These phases can be seen as arrays of polar/apolar interfaces, with separations on the order of molecular lengths. The

(*) Present address : Laboratoire de Physique des Solides, associé au CNRS (LA 2). Bâtiment 510, Université Paris Sud, 91405 Orsay, France.

$\left(^{+}\right)$Present address: D.P.C.-S.C.M.-UA 331, CEN Saclay, 91191 Gif sur Yvette, France. addition of water, which hydrates the polar heads, changes the relative volumes of the polar and apolar regions, and also their shapes. This results in a succession of lyotropic mesophases, which have been classified according to the shapes and repetitions of their interfaces [1-4]. The main structures are built by packing aggregates, whose surfaces have a uniform curvature : flat bilayers (one dimensional stacking, lamellar phase), circular cylinders (two dimensional packing, hexagonal phases), or objects with saddle-splay curvature (three dimensional, cubic phases). 
This leads to the classical picture of water/surfactant systems, with only two or three mesophases separated by large two-phase regions. In a way, this picture is quite surprising, as it implies that such a complex system is not capable of adjusting its structure in response to the main structural parameter (the water/surfactant ratio).

In fact, many more phases are known. A remarkable example is the egg lecithin/water system where Luzzati et al. [5] have shown that many phases can exist at different values of the temperature and composition (lamellar phases $\mathrm{L}_{\alpha}, \mathrm{L}_{\beta}, \mathrm{L}_{\delta}$; hexagonal phase : $\mathrm{H}$; rectangular phase : $P_{\delta}$; rhomboedral phase $R$ and cubic phase $Q)$. Similarly for potassium palmitate, four intermediate mesophases have been identified (deformed hexagonal, rectangular, complex hexagonal and cubic phase) [1]. All it takes to see them is an X-ray experiment capable of showing more than two diffraction lines and a sample preparation routine where the external parameter (composition, temperature) is scanned at sufficiently small intervals. Then the situation soon becomes quite complex, with no obvious relation between the nature of a given surfactant and the symmetries of the phases which it will produce.

For completeness let us also mention the variety of phases which are two-dimensional arrays of long stiff rods (deformed hexagonal or rectangular lattices $[1,2,5,6,7]$ the three dimensional networks with rhomboedral or tetragonal symmetry (phases R and $\mathrm{T}[8]$ ), or those with cubic symmetry (Ia3d, Pn3m, Pm3n, Im3m, Fd3m and $\mathrm{P}_{3} 32$ phases) [9-12].

All these phases occur at compositions between those of the hexagonal (cylindrical) and lamellar phase - hence their generic name intermediate [1, 13-15]. Then the following question arises : do they result from peculiar features of this or that surfactant system, or do they represent a general way for all surfactant/water systems to cross from cylindrical to planar geometry in response to a continuous variation of the polar and apolar volumes?

In the present work we examine the structures of the intermediate mesophases in the SDS/water system; the use of a high flux of X-rays allows us to extract more information from the scattering pattern than was previously available. At this level of resolution, we observe that the geometry of the aggregates changes nearly continuously from the most hydrated mesophase (hexagonal array of cylinders) to the least hydrated one (flat-bilayers).

The changes which we have observed occur through a deformation of the cylinders (hexagonal phase) into ribbons (2 dimensional monoclinic. phase), which then merge two by two sideways (period doubling) and also at regular intervals along their length (3 dimensional rhomboedral phase). A continuous deformation of this phase leads to an optically isotropic phase (cubic phase); the cubic structure may correspond to one of the three minimal surfaces defined mathematically by Schwarz [16], which had not been found experimentally until quite recently [12]. Then the cubic phase is stratified into separate layers which are piled in a staggered configuration (tetragonal phase). Finally the three dimensional correlations between these layers are lost, yielding a lamellar phase whose layers still contain a collection of defects with the short range correlations of the tetragonal phase [20].

At this point it is appropriate to precise our definition of phases: we identify a phase in the thermodynamic sense, by the fact that it will not mix with another phase. This is somewhat different from the traditional acceptation of the word phase in amphiphilic system, where all materials with the same structure would be labelled as members of a particular phase (for instance the hexagonal phase) regardless of whether or not they would be miscible.

\section{Methods.}

2.1 MATERIALS. - The purity (or lack of it) of the amphiphiles can have important consequences on the phase diagrams of the mesophases $[17,18]$. This is especially true for intermediate mesophases which exist only in narrow ranges of composition : such phases can be mixed completely if the main amphiphile is contamined with weak amphiphiles (e.g. long chain alcohols) or with salts. Most samples used in this work were made with SDS which was purchased from BDH (grade specially pure); this material was reneutralized to remove acid impurities [19] and dried at $65^{\circ} \mathrm{C}$ in a vacuum during 48 hours. This procedure ensures a good stability of the mesophases for a few days at $65^{\circ} \mathrm{C}$ and a few hours at $90^{\circ} \mathrm{C}$. For the sake of consistency with neutron scattering and NMR experiments $[19,20]$, the mesophases were made with $\mathrm{D}_{2} \mathrm{O}(99.99 \%$ isotopically pure from ORIS-BIS); these mesophases are identical with those made with $\mathrm{H}_{2} \mathrm{O}$ provided that identical mole fractions of water are used. Some samples were made with a SDS given to us by G. J. T. Tiddy of Unilever. They gave the same set of structures, but with slight differences in the locations of the concentration and temperature transitions between the mesophases. Finally, the procedures used for preparing the mesophases and assessing their homogeneity are identical to those previously described $[19,20]$.

2.2 SAMPLES. - Some samples were made in the usual way, with fixed compositions, in order to obtain a rough assessment for the localisations of the phase boundaries. The accuracy on the compositions of such samples was $\pm 1 \%$. Errors in these compositions are caused by gain or loss of water during the storage of «dry » SDS, during the mixing of SDS 
with $\mathrm{D}_{2} \mathrm{O}$, and while filling the $\mathrm{X}$-ray cells ; these errors are unavoidable unless the whole preparation is performed in a closed unit, where the water vapor pressure equals that of the desired mesophase.

Such errors are tolerable for systems where the mesophases have a wide extension in composition, such as non ionics or short chain ionic amphiphiles. However long chain ionic amphiphiles can produce a large number of different phases in a narrow composition range; in a temperature/composition phase diagram, the phase boundaries are nearly vertical, and separated by intervals on the order of $1 \%[1,14$, 21]. For such phase diagrams, the usual method of making samples at fixed compositions and scanning the temperature scale is clearly inappropriate : composition is the parameter which should be scanned.

To solve this problem, we deliberately made samples with a spatial gradient in their composition. This technique is derived from the classical water penetration methods, which are widely used for observing the textures of lyotropic mesophases under the microscope $[22,23]$; it is also quite similar to the capillary technique developped by R. G. Laughlin, for the determination of phase diagrams through a measurement of the refractive indexes across a composition gradient [24].

For our X-rays experiments we use a long rectangular cell of lateral dimensions $60 \times 5 \mathrm{~mm}$ and thickness $1.5 \mathrm{~mm}$ with mica windows; this assembly is held through additional teflon spacers in a two piece stainless steel holder, with stainless steel screws every $5 \mathrm{~mm}$; it is water tight if properly closed. Most of the cell's volume is filled with a water poor mesophase (in our case the lamellar phase of the SDS/water system), which is driven to one end of the cell through centrifugation. Then water is introduced at the opposite end of the cell through a pin hole which is subsequently plugged. The relative amounts of water and mesophase are calculated to match the central composition of the concentration range to be studied $(*)$.

The cell is then heated to a temperature where the mutual diffusion of water and amphiphile molecules is sufficiently rapid (in our case $55^{\circ} \mathrm{C}$ ). In a few hours, this diffusion produces a complete sequence of phases ; these can be seen with the naked eye as separate bands with discontinuities of refractive index at their boundaries; the whole sequence is a few $\mathrm{mm}$ long. This pattern spreads very slowly in a few days; it can be considered as stable over the exposure times of our X-ray diffraction patterns (a few hours at LURE).

This gradient cell is mounted in an oven (basically

(*) Sometimes, the sequence of phases can be monitored by allowing water to evaporate from a concentrated surfactant solution. a large copper block : $10 \times 10 \times 10 \mathrm{~cm}$ ) which ensures a temperature homogeneity of $0.1{ }^{\circ} \mathrm{C}$ over the whole sample. This oven is placed in the $\mathrm{X}$-ray beam of the line D24 at LURE; it is translated with respect to the beam in order to explore the full succession of mesophases present in the cell. We use semi-punctual collimation $(300 \times 800 \mu \mathrm{m})$, with the smallest dimension of the beam along the composition gradient of the cell; this allows the diffraction pattern to be recorded at very short intervals $(\sim 300 \mu \mathrm{m})$ along the composition gradient. The corresponding resolution in water concentration is $\sim 0.05 \%$ in weight for a sample which has been aged for a few days.

The main advantage of this method is that it allows a systematic exploration of the phase diagram according to the composition variable at a constant temperature. The results are also more reproducible than with the preparation of samples of fixed composition, because errors in the total amount of water in the sample will only shift the pattern towards one end of the cell, and also because minute losses of water can be tolerated as long as they are small compared to the amounts of water transported by self diffusion along the concentration gradient.

Still, the method has two drawbacks. First, the correspondence between spatial scale and concentration scale is only approximate, and therefore the absolute location of the phase boundaries must be established through another method. Second, the samples must be rather thick $(1.5 \mathrm{~mm})$ in order to avoid the growth of large crystallites during the long annealing times. This large thickness may lead to a strong adsorption of the primary wavelength $(1.608 \AA)$ by the sample, and to a corresponding enhancement of the diffraction by subharmonics of this wavelength (mostly $\lambda / 3$, and also $\lambda / 4$ ).

2.3 Data Collection. - The diffraction patterns come out as Debye-Scherer rings produced by all domains in the irradiated volume. For the first diffraction orders, we used long sample to detector distances (up to $1308 \mathrm{~mm}$ ) in order to resolve overlapping rings; in this configuration the resolution was $\Delta Q=10^{-3} \AA^{-1}$ and the smallest $Q$ value near the edge of the beam stop was $Q_{\min }=8 \times$ $10^{-3} \AA^{-1}$ (with the scattering vector $Q$ defined as $(4 \pi / \lambda) \sin \theta$ where $2 \theta$ is the scattering angle). At larger angles, the weaker intensities of the higher orders and the geometry of the experiment forced us to use shorter sample to detector distances, down to $400 \mathrm{~mm}$; then the largest $Q$ value which could be reached was $0.55 \AA^{-1}$. This $Q$ range was adequate since the first diffraction lines of the intermediate phases start at $Q=0.06 \AA^{-1}$, and their higher orders become too weak to be observed beyond $Q=0.5 \AA^{-1}$.

As detectors, we used both photographic films 
and linear position sensitive detectors (PSD). Films have the advantages of covering a much larger area of reciprocal space, and at the same time offering a better resolution. Moreover, because they give a two-dimensional image, they still give a meaningful information when the diffraction pattern consists of spots instead of smooth rings, as may happen with some phases which easily grow large crystallites. Thus we found it necessary to always obtain a film from every phase before using the linear detector, in order to look for diffraction spots and to resolve poorly separated lines; once the structure was identified, the linear detector was used to determine the relative intensities of the diffraction lines and measure the variations of their positions through the composition range of a given phase.

\section{Results.}

The mesophases of SDS in water form only above room temperature because the hydrated crystals of SDS coexist with excess water up to $30^{\circ} \mathrm{C}$. Then the excess water is used to form mesophases where the SDS molecules are in a liquid state [1, 17, 21]. In a diffraction experiment these mesophases are easily told apart from the hydrated crystals : their wide angle scattering shows a diffuse ring located at $2 \pi / 4.5=1.4 \AA^{-1}$ which is produced by the distances between neighbouring SDS chains within an aggregate.

The first mesophase to be formed, at $30^{\circ} \mathrm{C}$, is the most hydrated one : the hexagonal phase. Then, as the temperature rises above $40{ }^{\circ} \mathrm{C}$, other, less hydrated, mesophases may appear, depending on the sample's composition. The last mesophase to appear (in a temperature scan) is the lamellar phase whose lowest point is at $50{ }^{\circ} \mathrm{C}$.

Here we present the results obtained at a fixed temperature $\left(55^{\circ} \mathrm{C}\right)$, in a scan of the water/SDS concentrations.

3.1 Hexagonal PHASE $\left(\mathrm{H}_{\alpha}\right)$. - This phase is observed in the composition range $38 \%$ SDS to $57.1 \%$ (as expressed in weight of SDS/weight of $\mathrm{D}_{2} \mathrm{O}$ ). It is characterized by a diffraction pattern which only contains 3 or 4 sharp lines at small angles with spacings in the ratios $1, \sqrt{3}, \sqrt{4}, \sqrt{7}$. The unit cell in real space is a parallelogram whose edges are all equal and whose angle is $120^{\circ}$; at $T=55^{\circ} \mathrm{C}$ and SDS content $49.8 \%$, the edge length $a$ is $47.4 \AA$.

Near the upper composition boundary of the phase, the profile of the diffraction lines becomes broader than the experimental resolution (Fig. 1). This profile indicates the presence of a diffuse band located on the external side of the diffraction line. Remarkably, the transition to the next mesophase is nearly continuous : at $55^{\circ} \mathrm{C}$ the width of the two phase region is only $0.2 \%$ in composition, and at $70{ }^{\circ} \mathrm{C}$ we cannot find a two-phase region. Therefore,

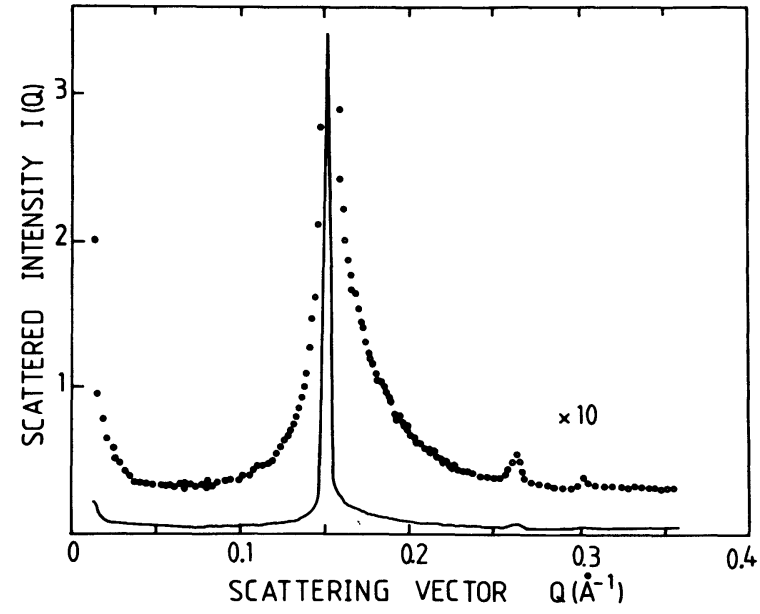

Fig. 1. - Diffraction lines from a hexagonal mesophase at $56 \%$ SDS and $T=55^{\circ} \mathrm{C}$; the data are obtained by recording a powder pattern with a linear PSD. The 3 diffraction lines have spacings in the ratios $1, \sqrt{3}$, $\sqrt{4}$, with the first order located at $Q=0.152 \AA^{-1}$. In real space the unit cell is a parallelogram whose edges are equal to $a=47.7 \AA$ at an angle of $120^{\circ}$. There is also a substantial amount of diffuse scattering on the external side of the first diffraction line; this is caused by the proximity of the upper composition boundary for the hexagonal phase, which is located at $57.1 \%$ SDS.

the diffuse band which appears near this composition may be produced by a soft mode associated with the transition.

3.2 TWO-DIMENSIONAL MONOCLINIC PHASE $\left(\mathrm{M}_{\alpha}\right)$. - This phase is characterized by a diffraction pattern containing seven sharp lines below $Q=0.55 \AA^{-1}$ (Fig. 2a). Only the two first are strong; all the followings ones are rather weak. The widths of these lines are equal to the experimental resolution $\left(\Delta Q=10^{-3} \AA^{-1}\right)$, except for the first line (Fig. 2b), which is very broad at low temperature $\left(\sim 40^{\circ} \mathrm{C}\right)$, and sharper at higher temperatures. These spectra can be reproduced with a two-dimensional monoclinic lattice (Tab. I) : then the observed lines are indexed as (10); (01); (11); (20); $(1 \overline{2}) ;(02)$ and $(30)$. In real space the unit cell can be considered as a deformation of the parallelogram (or regular hexagon) of the hexagonal phase. Within this phase, the unit cell is progressively deformed : one of the edges becomes longer than the other and the angle between them decreases continuously. These results are in a good agreement with the observations of R. M. Wood et al. [25].

This structure is not unique to the SDS/water system : it has also been observed in soap/water systems by Luzzati et al. [1] and called deformed hexagonal; however in that case the angle between the unit vectors of the unit cell was not determined (because only h0 and $0 \mathrm{k}$ diffraction lines were observed). 


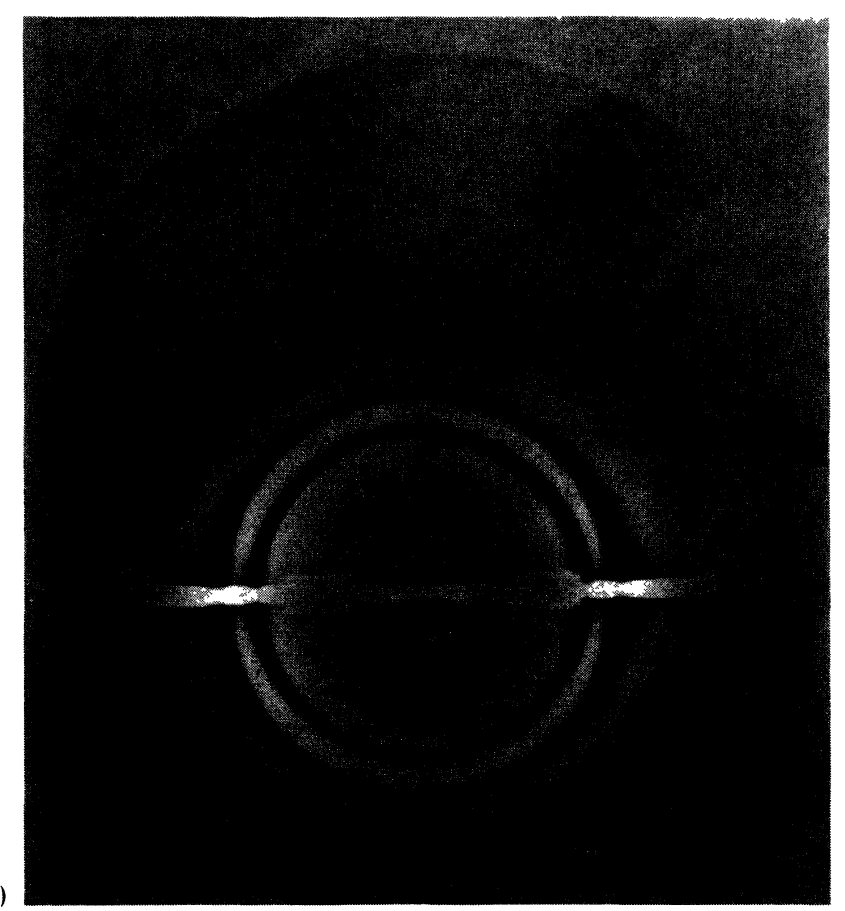

b)

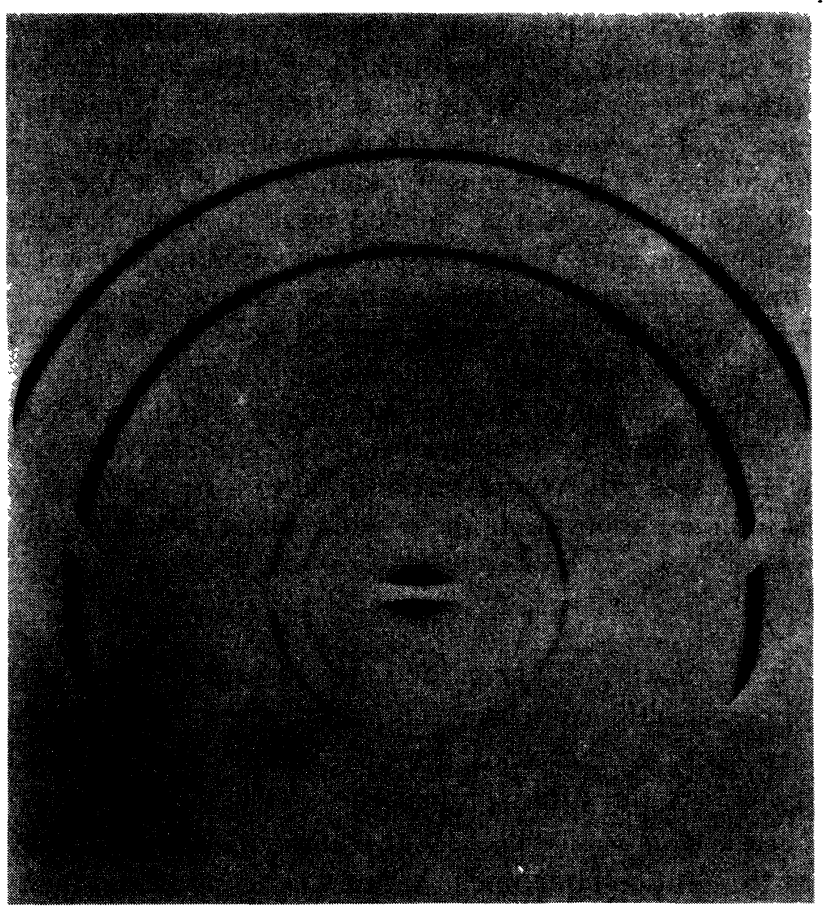

Fig. 2. - Powder diffraction pattern from a point located within the concentration gradient sample. The composition at this point matches that of the first intermediate mesophase $\mathrm{M}_{\alpha}\left(\mathrm{SDS} \sim 60 \% ; T=55^{\circ} \mathrm{C}\right)$. (a) Sample to film distance $=519 \mathrm{~mm}$; the first two lines close to the beam are produced by a subharmonic of the main wavelength, the two intense ones are (10) and (01) and the next are $(11) ;(20) ;(1 \overline{2}) ;(02) ;(30)$ (see Tab. I). (b) Sample to film distance ; $1122 \mathrm{~mm}$; the second intense line (01) has a width controlled by the experimental resolution, while the other one (10) is somewhat broader. The unit cell is a deformation of the regular hexagon of the hexagonal phase : the edge lengths are $a=48.8 \AA$ and $c=39.3 \AA$ making an angle $\beta=114.1^{\circ}$.
Table I. $-Q_{\mathrm{obs}}$ and $Q_{\mathrm{cal}}$ are the scattering vectors $\left(Q=\frac{4 \pi}{\lambda} \sin \theta\right)$ of the observed reflexions (see Fig. 2a), and calculated for the two-dimensional monoclinic phase with $a=48.8 \AA, c=39.3 \AA$ and $\beta=114.1^{\circ}$. The observed intensities were visually estimated (vvs : extremely strong; vs : very strong; $s$ : strong ; $m:$ medium ; $w$ : weak; $v w$ : very weak; $v v w$ : extremely weak). The two-lines observed close to the beam stop are produced by a subharmonic of the wavelength. They are not reported in the table.

\begin{tabular}{c|c|c|c|c}
\hline$h k$ & $\begin{array}{c}Q_{\text {obs }} \\
\left(\AA^{-1}\right)\end{array}$ & $\begin{array}{c}Q_{\text {cal }} \\
\left(\AA^{-1}\right)\end{array}$ & $\begin{array}{c}\text { Error } \\
(\%)\end{array}$ & $\begin{array}{c}\text { Intensity } \\
\text { observed }\end{array}$ \\
\hline $1 \frac{0}{1}$ & 0.1411 & 0.1410 & 0.07 & vs \\
$0 \frac{1}{1}$ & 0.1751 & 0.1752 & 0.06 & vvs \\
11 & & 0.2643 & & \\
20 & 0.2664 & 0.2660 & 0.15 & w \\
$1 \frac{2}{2}$ & 0.3188 & 0.2820 & 0.21 & $\mathrm{~s}$ \\
$2 \frac{2}{2}$ & 0.3495 & 0.3198 & 0.31 & vvw \\
$0 \frac{2}{31}$ & & 0.3504 & 0.23 & vw \\
21 & & 0.3861 & & \\
$3 \frac{0}{32}$ & 0.4240 & 0.3880 & & \\
12 & & 0.4230 & 0.24 & \\
\hline
\end{tabular}

3.3 THREE DIMENSIONAL RHOMBOEDRAL PHASE $\left(R_{\alpha}\right)$. - The existence of this phase in the SDS/water system had not been recognized previously. Its structure is the same as that of the $R$ phase observed in anhydrous strontium soaps and egg lecithin [5, 8]. Figure 3 presents some films obtained from non oriented samples prepared in this phase. Up to 8 diffraction lines can be seen, and their positions in the film are given in table II ; all of them are sharp, excepted for the fifth and the sixth one away from the beam. Moreover, between these two lines a diffuse band can be observed. The diffraction patterns can be indexed on a rhomboedral lattice, or on a less symmetric lattice : a face centred orthorhombic. The fit to our diffraction patterns is very good, with either one of these solutions (Tab. II).

This phase extends approximately from $62.3 \%$ SDS to $64.8 \%$ SDS. Throughout this range, it undergoes a remarkable deformation which brings it very close to either of the surrounding phases.

For the rhomboedral solution to the diffraction patterns, we find that the edge $a$ and the angle $\alpha$ remain roughly constant through most of the concentration range, excepted in the vicinity of the lower concentration boundary (next to $\mathbf{M}_{\alpha}$ ) where $a$ stretches and $\alpha$ decreases rapidly as water is added (Fig. 4). 


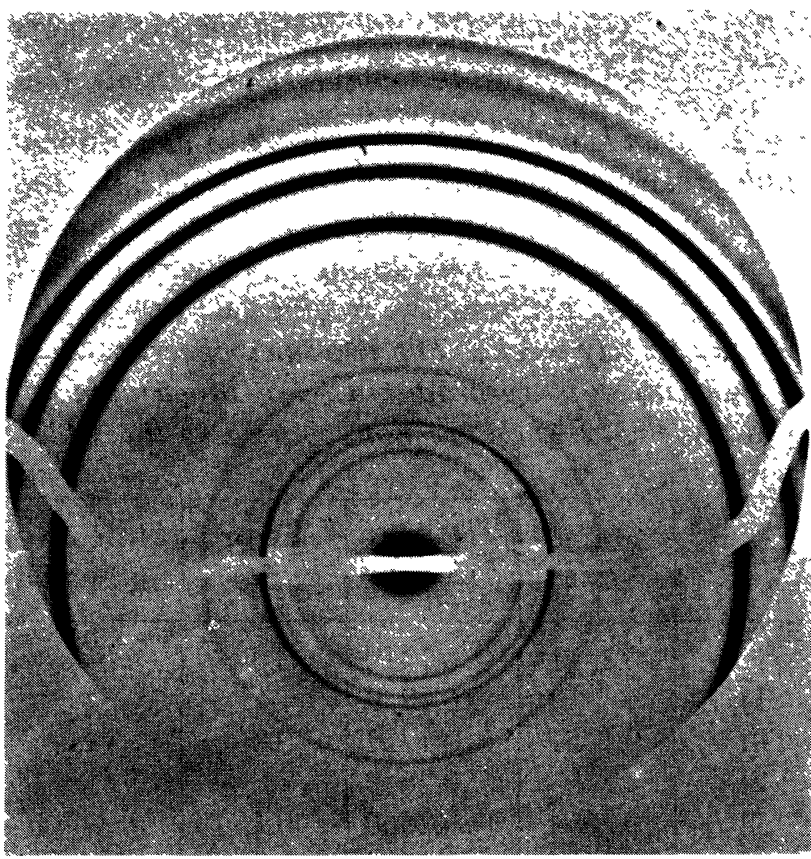

Fig. 3. - Powder diffraction pattern from a point located at $x=6.2 \mathrm{~mm}$ in the concentration gradient sample (see Fig. 4). This spectrum can be indexed either on a rhomboedral lattice or on a face-centred orthorhombic one (see Tab. II). The long period of this lattice comes from the position of the first diffraction line at $Q=0.0776 \AA^{-1}$, which is much closer to the beam than for the previous 2dimensional monoclinic phase. Note that some lines are broader than the experimental resolution; also the diffuse band located between the 5th and 6th lines is so far unexplained. On the figure one centimeter corresponds to $\sim 0.04 \AA^{-1}$.

For the associated triple hexagonal cell (which is equivalent to the primitive rhomboedron) the edge $a^{\prime}=2 a \sin \frac{\alpha}{2}$ of the basis hexagon remains roughly constant, while the edge $c^{\prime}=a \sqrt{3} \sqrt{1+2 \cos \alpha}$ in the $Z$-direction stretches as water is added to approach the two dimensional monoclinic $\mathbf{M}_{\alpha}$ phase. If $c^{\prime}$ were to become infinite, then the transition to a two-dimensional monoclinic phase with an angle $\beta=120^{\circ}$ would be continuous. In fact there is a discontinuity and at the boundary we find $a^{\prime}=167$ $\AA$ and $c^{\prime}=133 \AA$.

When water is removed to reach the other end of the phase, the angle $\alpha$ increases to $109.47^{\circ}$ and $a$ shrinks to $100.6 \AA$ : at this point the symmetry of the lattice becomes cubic. However we find a very slight discontinuity at this transition, corresponding to a jump $\Delta a=0.1 \AA$ or $\Delta \alpha=0.05^{\circ}$ (Fig. 4).

For the face-centred orthorhombic as a solution to the diffraction patterns, the law of the deformation would be given by a relation between the edges of the unit cell, which remain valid throughout the entire range of this phase.

$$
|\mathbf{c}|=\frac{a b}{\sqrt{3 a^{2}-b^{2}}} .
$$

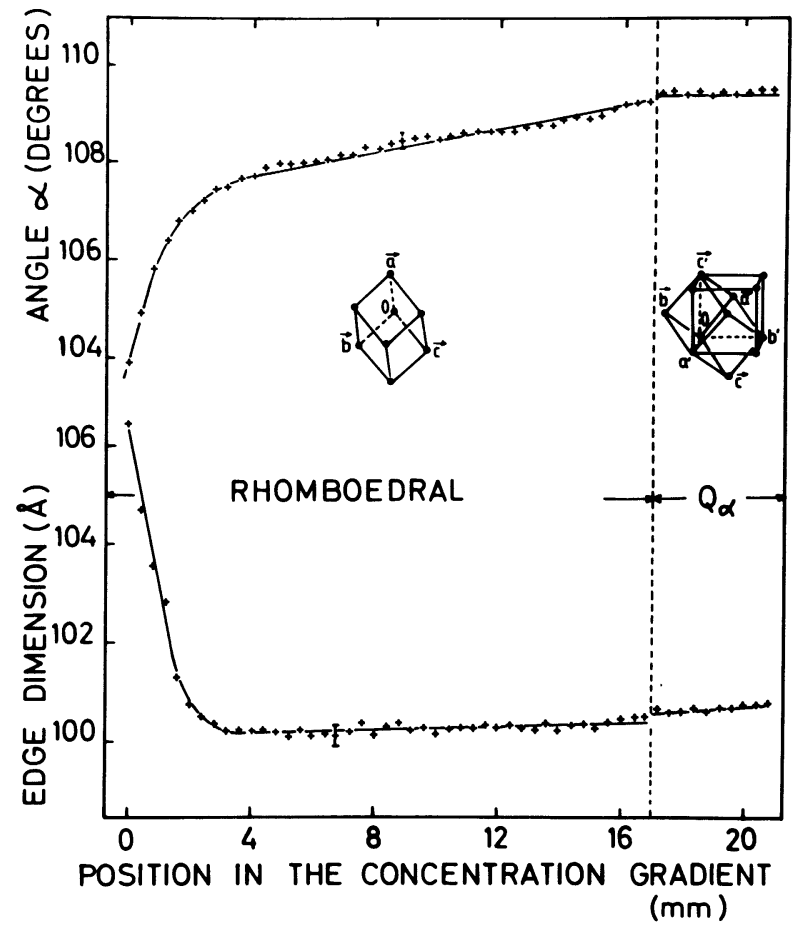

Fig. 4. - Transition from the rhomboedral phase $\mathbf{R}_{\alpha}$ to the cubic phase $Q_{\alpha}$. The diffraction patterns are recorded with a linear position sensitive detector at very short intervals $(\sim 300 \mu \mathrm{m})$ along the composition gradient; the figure shows the edge length $a$ and the angle $\alpha$ of the unit cell as functions of the sampled position in the concentration gradient. The corresponding resolution in water concentration is $-0.05 \%$ in weight and the composition range explored here extends from SDS $\sim 62.3 \%$ to SDS $\sim 65.4 \%$. At the higher limit, the angle $\alpha$ locks at $109.48^{\circ}$ which is the value for the cubic phase; at lower SDS concentration, the structure becomes more anisotropic as $\alpha$ decreases ; the complete transition to a structure made of infinite ribbons (as in the previous phase) would require $\alpha=0$.

Then, as water is added to approach the two dimensional monoclinic phase $\mathbf{M}_{\alpha}, a$ remains roughly constant, $c$ increases rapidly while $b$ shrinks to $a \sqrt{3}$. At the other end of the phase, both $a$ and $b$ shrink to $a=b=c \sqrt{2}$; at this point the symmetry of the unit cell becomes that of a centred cubic body.

Of course, one could index the same spectra on a less symmetric lattice. This would involved a large number of structure parameters for the description of the unit cell ; but then, these parameters would have to be tied by a relation, which would reduce the number of independant parameters.

3.4 CUBIC PHASE $\left(\mathrm{Q}_{\alpha}\right)$. - Like the previous one, this phase had not been identified previously in SDS. Figure 5 shows a diffraction pattern of this phase. Up to 14 diffraction lines can be seen. The spacings of all the observed reflexions are in perfect agreement with the reflexions of a cubic lattice with the indices given in the table III. The $Q$ values of the 
Table II. - The diffraction pattern shown in figure 3 can be indexed either on a rhomboedral lattice or on a facecentred orthorhombic lattice. Both solutions fit our spectra quite accurately. The rhomboedral unit cell is defined by $a=99.9 \AA$ and $\alpha=108.3^{\circ}$ in real space, or $a^{*}=0.0746 \AA^{-1}$ and $\alpha^{*}=62.7^{\circ}$ in reciprocal space. An assignment to an orthorhombic symmetry would also be possible, but the edges of the unit cell would then be have tied by the relation $c^{*}=\sqrt{3 b^{*^{2}}-a^{*^{2}}}$ in reciprocal space. The orthorhombic unit cell would have edges $a^{*}=0.0418 \AA^{-1}$, $b^{*}=0.0387 \AA^{-1}$ and $c^{*}=0.0524 \AA^{-1}$ or, $a=150.2 \AA, b=162.2 \AA$ and $c=119.8 \AA$ in real space.

\begin{tabular}{|c|c|c|c|c|c|c|c|}
\hline \multicolumn{3}{|c|}{ Rhomboedral lattice } & \multicolumn{2}{|c|}{ Experimental } & \multicolumn{3}{|c|}{ Orthorhombic lattice } \\
\hline $\begin{array}{c}h k l \\
h, k, l \\
\text { permutable }\end{array}$ & $\begin{array}{c}Q_{\mathrm{cal}} \\
\left(\AA^{-1}\right)\end{array}$ & $\begin{array}{l}\text { error } \\
(\%)\end{array}$ & $\underset{\left(\AA^{-1}\right)}{Q_{\text {obs }}}$ & $\begin{array}{l}\text { Intensity } \\
\text { observed }\end{array}$ & $\begin{array}{c}h k l \\
h+k ; k+l ; l+h=2 n\end{array}$ & $\underset{\left(\AA^{-1}\right)}{Q_{\text {cal }}}$ & $\begin{array}{l}\text { Error } \\
(\%)\end{array}$ \\
\hline 100 & 0.0746 & 0.00 & & & & & \\
\hline $1 \overline{1} 0$ & 0.0776 & 0.00 & 0.0776 & $\mathrm{w}$ & $\begin{array}{l}020 ; 111 \\
200\end{array}$ & $\begin{array}{l}0.0775 \\
0.0836\end{array}$ & 0.13 \\
\hline $11 \overline{1}$ & 0.1076 & & & & 002 & 0.1049 & \\
\hline 110 & 0.1274 & & & & 220 & 0.1140 & \\
\hline $20 \overline{1}$ & 0.1327 & & & & 022 & 0.1304 & \\
\hline$\overline{2} 11$ & 0.1344 & 0.07 & 0.1345 & vvs & $202 ; 131$ & 0.1342 & 0.22 \\
\hline $2 \underline{00}$ & 0.1492 & & & & 311 & 0.1414 & \\
\hline $2 \overline{2} 0$ & 0.1552 & 0.06 & 0.1553 & vs & $040 ; 222$ & 0.1549 & 0.26 \\
\hline $21 \overline{1}$ & 0.1682 & 0.24 & 0.1678 & $\mathbf{s}$ & 400 & 0.1673 & 0.30 \\
\hline $2 \overline{2} 1$ & 0.1722 & & & & 240 & 0.1760 & \\
\hline 111 & 0.1789 & 0.06 & $\begin{array}{l}0.1790 \\
\text { (large) }\end{array}$ & $\mathrm{w}$ & $\left\{\begin{array}{l}331 \\
420\end{array}\right.$ & 0.1789 & 0.06 \\
\hline $3 \overline{11}$ & 0.1889 & 1.27 & $\sim 0.1865$ & $\mathrm{vw}$ & 042 & 0.1843 & 1.16 \\
\hline & & & (diffuse) & & 402 & 0.1871 & 0.32 \\
\hline 120 & 0.1950 & 0.10 & 0.1952 & $\mathrm{w}$ & & 0.1974 & 1.13 \\
\hline $30 \overline{1}$ & 0.2008 & & & & 133 & 0.2005 & \\
\hline$\underline{3} 0 \overline{2}$ & 0.2042 & & & & & & \\
\hline$\overline{3} 21$ & 0.2053 & 0.24 & 0.2058 & $\mathrm{w}$ & $242 ; 151 ; 313$ & 0.2049 & 0.44 \\
\hline
\end{tabular}

lines are given by $Q=\frac{2 \pi}{a}\left(h^{2}+k^{2}+\ell^{2}\right)^{-1 / 2}$ where $a$ is equal to $116 \AA$ at $55^{\circ} \mathrm{C}$ and $h+k+\ell$ is an even integer. Because we observe so many lines, we can do better than just determine the shape and dimensions of the unit cell. From the existence or extinction of certain lines we can try to specify the space group which describes the symmetry of this unit cell, and from there discuss the locations of surfactant aggregates within this cell.

The three simplest types of unit cells are primitive (simple cubic), body centred, and face centred. We know that the lattice cannot be face centred, because the ratios of the first observed lines $(1, \sqrt{2}$, $\sqrt{3}, \sqrt{4}, \sqrt{5}, \sqrt{6}, \sqrt{7}$ ) differ from those of a lattice with a face centred unit cell $(1, \sqrt{4 / 3}, \sqrt{8 / 3}$, $\sqrt{11 / 3}, \sqrt{12 / 3}, \sqrt{16 / 3}, \ldots)$. Moreover, since all observed reflexions have Miller indices with $h+$ $k+\ell=2 n$, we assume that the lattice is body centred rather than primitive : otherwise it would be necessary to invoke a very odd form factor to cancel all the lines with $h+k+\ell=2 n+1$.

JOURNAL DE PHYSIQUE. - T. 48, N* 9, SEPTEMBRE 1987
Next, we consider the space group. The observation of diffraction lines with indices $h^{2}+k^{2}+\ell^{2}$ equal to $2,4,10,12$ or 18 excludes the usual space groupe Ia3d. In the same manner, the lines at $h^{2}+k^{2}+\ell^{2}=2,4,12$ and 18 rule out the space group $\mathrm{I} \overline{4} 3 \mathrm{~d}$, and those at $h^{2}+k^{2}+\ell^{2}=2$ or 10 rule out Ia3 ; finally the observation of a weak line at $h^{2}+k^{2}+\ell^{2}=4$ is also incompatible with 14,32 . In the end, 6 possible space groups remain: $\operatorname{Im} 3 \mathrm{~m}$; $\mathrm{I} 432 ; \mathrm{I} \overline{4} 3 \mathrm{~m} ; \mathrm{Im} 3 ; \mathrm{I} 23 ; \mathrm{I} 23$. Among these possible space groups, the $\operatorname{Im} 3 \mathrm{~m}$ is remarkable because it matches the symmetry of one of the three minimal surfaces of Schwarz [16].

A phase with this space group has been reported recently in a ternary system [12] $\left({ }^{*}\right)$.

(*) Note that this space group Im $3 \mathrm{~m}$ had been originally proposed as a possible candidate in the glycerol monooleate/water system [26] ; however in that case, a better understanding of the phase diagram has shown that the assignment of X-ray lines was incorrect [27]. 


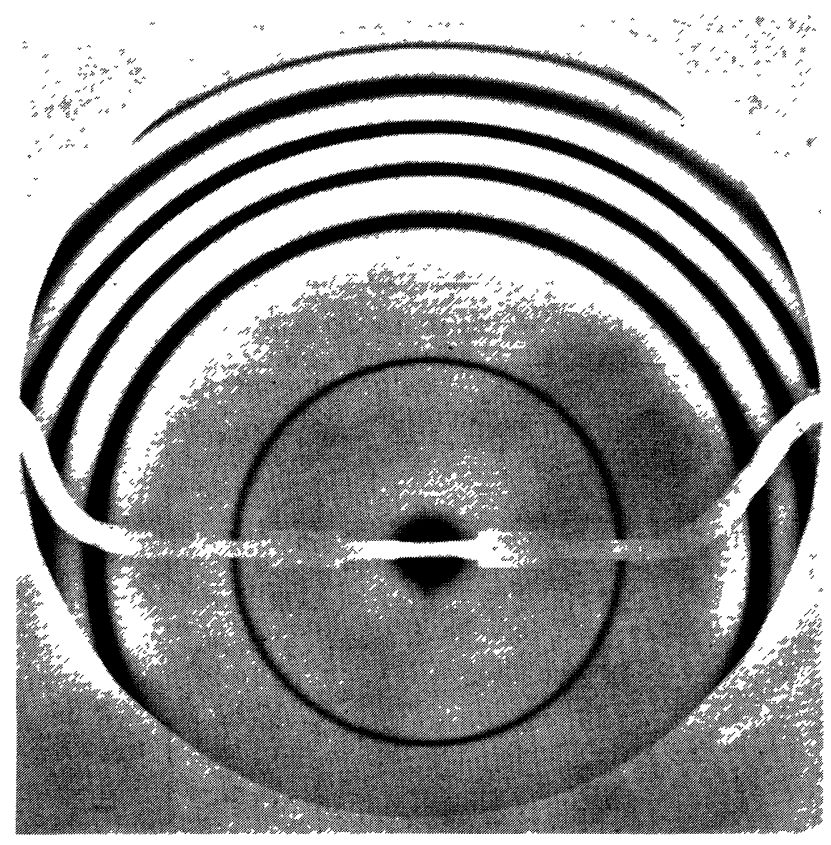

(l)

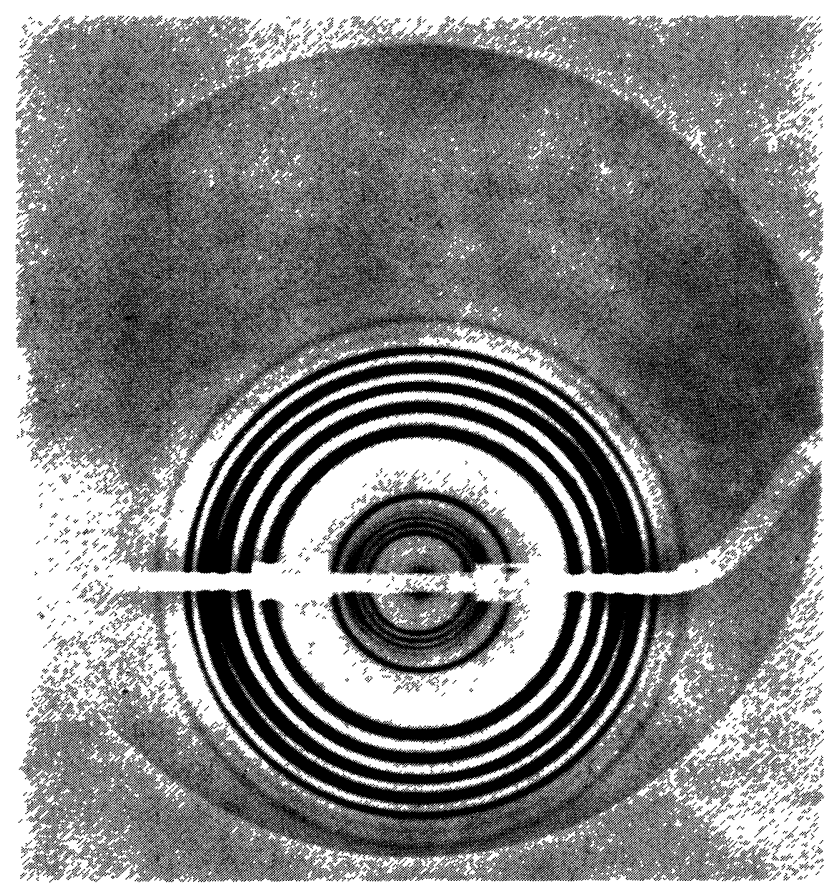

b)

Fig. 5. - Powder diffraction patterns from samples in the cubic phase $Q_{\alpha}$. (a) Sample made in the usual way at a fixed, uniform composition (SDS $65.0 \%$; sample thickness $0.7 \mathrm{~mm}$; sample to film distance $(1128 \mathrm{~mm})$. The film was overexposed in order to show the higher diffraction orders ; note that the sixth line (222) is broader than the experimental resolution. (b) Sample with a concentration gradient; the beam hits the point located at $x=20.0 \mathrm{~mm}$. High intensities are obtained because of a shorter sample to film distance $(518 \mathrm{~mm})$ and long exposure : 14 diffraction lines are observed (Tab. III). In addition, scattering by the subharmonics of the main wavelength gives a set of 8 lines closer to the beam; this scattering is substantial because the gradient sample is rather thick $(1.5 \mathrm{~mm})$.
3.5 Tetragonal PHase $\left(\mathrm{T}_{\alpha}\right)$. - This phase has a very narrow range of existence between the $\mathrm{Q}_{\alpha}$ and $\mathrm{L}_{\alpha}$ phases (less than $0.6 \%$ in water/SDS concentration at $55^{\circ} \mathrm{C}$ ). It had not been reported previously for SDS/water presumably because it is so narrow, and also because it gives only 3 intense diffraction lines, which are close to some lines of the $Q_{\alpha}$ and $\mathrm{L}_{\alpha}$ phases ; thus if a linear position sensitive detector is used, it is possible to mistake this phase for a mixture of $\mathrm{Q}_{\alpha}$ and $\mathrm{L}_{\alpha}$.

With photographic film, the $T_{\alpha}$ phase is easily recognized through its texture, which is an array of large crystallites (much larger than in any of the other phases, which give smooth powder rings) (Fig. 6). Moreover, with high flux and high resolution a number of weak lines show up in addition to the 3 main lines : with a 13 hour exposure at LURE, and $\Delta Q / Q=10^{-3} \AA^{-1}$ we obtain 12 diffraction lines (Tab. IV).

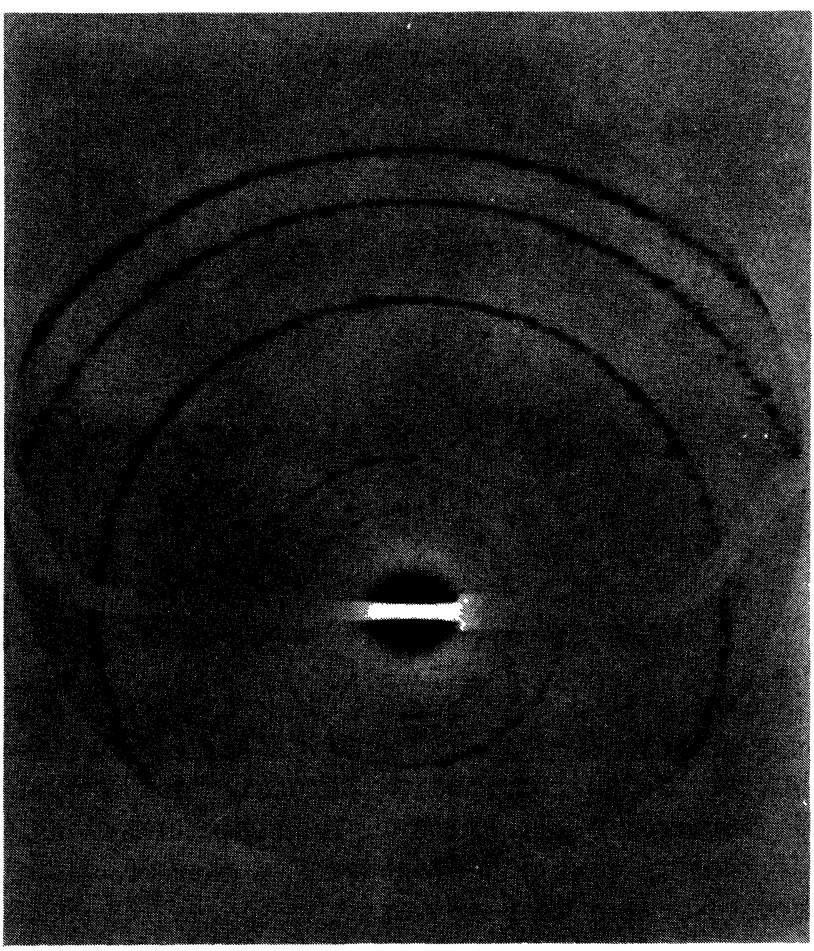

Fig. 6. - Diffraction pattern from a point located in the concentration gradient sample. The composition at this point matches that of the last intermediate mesophase $\mathrm{T}_{\alpha}$ (SDS $\sim 65.9 \% ; T=55^{\circ} \mathrm{C}$; sample to film distance $1128 \mathrm{~mm}$ ). The granular texture of the pattern indicates that the sample is made of many large, disoriented crystallites. To some extent, smoother diffraction rings can be obtained if the sample is quenched from a high temperature and reheated to $55^{\circ} \mathrm{C}$; the positions of the lines remain the same. Here 3 diffraction lines are observed after a one hour exposure (the subharmonics at low $Q$ are discarded) ; with longer exposures (13 hours at LURE), another 9 weak lines are obtained. These lines are indexed as $(101) ;(200)$ and (002) yielding a unit cell of dimensions $a=b=76.1 \AA$ and $c=67.2 \AA$. 
Table III. - In addition to the first lines close to the beam stop, which are produced by a subharmonic of the wavelength, the diffraction lines of the figure 5 are generated by a body-centred cubic lattice $\left(a^{*}=0.0538 \AA^{-1}\right.$; $a=116.8 \AA$ ).

\begin{tabular}{|c|c|c|c|c|c|}
\hline $\begin{array}{c}h k l \\
h, k, l \text { permutable } \\
(h+k+l=2 n)\end{array}$ & $h^{2}+k^{2}+l^{2}$ & $Q_{\text {obs }}\left(\AA^{-1}\right)$ & $Q_{\text {cal }}\left(\AA^{-1}\right)$ & Error $(\%)$ & $\begin{array}{l}\text { Intensity } \\
\text { observed }\end{array}$ \\
\hline $\left.\begin{array}{l}110 \\
200 \\
211 \\
220 \\
310 \\
222 \\
321 \\
400 \\
411 \\
330 \\
420 \\
332 \\
422 \\
510 \\
431 \\
521 \\
440 \\
530 \\
433 \\
600 \\
442 \\
611 \\
532\end{array}\right\}$ & $\begin{array}{r}2 \\
4 \\
6 \\
8 \\
10 \\
12 \\
14 \\
16 \\
18 \\
20 \\
22 \\
24 \\
26 \\
30 \\
32 \\
34 \\
\\
36 \\
\\
38 \\
40 \\
42 \\
44 \\
46 \\
48 \\
\\
50\end{array}$ & $\begin{array}{r}0.0759 \\
\sim 0.1070 \\
0.1319 \\
0.1522 \\
0.1706 \\
0.1865 \\
0.2014 \\
0.2154 \\
0.2280 \\
0.2427 \\
\\
0.2642 \\
0.2750 \\
\end{array}$ & $\begin{array}{l}0.0761 \\
0.1076 \\
0.1318 \\
0.1522 \\
0.1702 \\
0.1864 \\
0.2013 \\
0.2152 \\
0.2283 \\
0.2406 \\
0.2524 \\
0.2636 \\
0.2744 \\
0.2947 \\
0.3044 \\
0.3138 \\
\\
0.3229 \\
\\
0.3317 \\
0.3403 \\
0.3487 \\
0.3569 \\
0.3650 \\
0.3728 \\
0.3805\end{array}$ & $\begin{array}{l}0.26 \\
0.56 \\
0.08 \\
0.00 \\
0.23 \\
0.05 \\
0.05 \\
0.09 \\
0.13 \\
0.87 \\
0.23 \\
\\
0.22 \\
\\
\\
\end{array}$ & $\begin{array}{c}\mathrm{w} \\
\mathrm{vvw} \\
\mathrm{vvs} \\
\mathrm{vvs} \\
\mathrm{vs} \\
\mathrm{s} \\
\mathrm{s} \\
\mathrm{w} \\
\mathrm{vw} \\
\mathrm{vvw} \\
\mathrm{vw} \\
\mathrm{w} \\
\mathrm{wvw}\end{array}$ \\
\hline
\end{tabular}

These spectra can be indexed on a three-dimensional tetragonal lattice with a centred unit cell $(h+k+\ell$ is an even integer for all lines). This unit cell is related to that of the previous phase : indeed the edge length $a$ of its square base is roughly equal to the half diagonal of the cube face while its height $c$ is equal to a third of the long diagonal of the cube.

Note that the form factor of this phase is such that a $2 \mathrm{~d}$ assignment would almost match its spectrum. Indeed the modulation of the electron density along the directions parallel to the square base of the unit cell is rather slight. This is an important feature as it indicates that the structure tends to become stratified in one direction, obviously a step towards the production of a genuine lamellar structure. Other oddities are the weak intensities of the lines with indices such as $h k 0$ or $h k l$ with $k \neq 0$ (planes other than the rectangular faces of the unit cell), and the location of the weak $h k 0$ lines next to the strong $h 0 l$ lines (because $c$ is not much larger than $a$ ). All these features could lead to an (incorrect) assignment of the spectra on a two dimensional rectangular structure.

A phase with the same diffraction pattern and presumably the same structure has been observed in anhydrous long chain calcium soaps by Luzzati $e t$ al. [8] and called «phase $T$ ».

3.6 Lamellar PHASE $\left(\mathrm{L}_{\alpha}\right)$. - In this phase, the scattering pattern is dominated by the diffraction lines corresponding to the one-dimensional stacking of the bilayers ; up to 4 orders are observed (spacings in the ratios $1,2,3,4)$, with the first order at $Q=0.183 \AA^{-1}$ for compositions near the lowest 
Table IV. - Assignment of the diffraction lines of the $T_{\alpha}$ phase on a body centred tetragonal lattice, with $a=b=76.1 \AA$ and $c=67.2 \AA\left(a^{*}=b^{*}=0.0826 \AA^{-1}\right.$ and $c^{*}=0.0935 \AA^{-1}$ in reciprocal space).

\begin{tabular}{|c|c|c|c|c|}
\hline $\begin{array}{c}h k l \\
h, k \\
\text { permutable } \\
h+k+l=2 n\end{array}$ & $\frac{Q_{\text {obs }}}{\left(\AA^{-1}\right)}$ & $\begin{array}{c}Q_{\mathrm{ca} 1} \\
\left(\AA^{-1}\right)\end{array}$ & $\begin{array}{c}\text { Error } \\
(\%)\end{array}$ & $\begin{array}{l}\text { Intensity } \\
\text { observed }\end{array}$ \\
\hline 110 & 0.1170 & 0.1168 & 0.17 & vvw \\
\hline 101 & 0.1249 & 0.1248 & 0.08 & vs \\
\hline 200 & 0.1651 & 0.1652 & 0.06 & vs \\
\hline 002 & 0.1869 & 0.1870 & 0.05 & vvs \\
\hline 211 & & 0.2070 & & \\
\hline 112 & $\sim 0.2210$ & 0.2205 & 0.01 & $\mathrm{vw}$ \\
\hline 220 & & 0.2336 & & \\
\hline 202 & 0.2488 & 0.2495 & 0.28 & $\mathrm{w}$ \\
\hline 310 & & 0.2612 & & \\
\hline 301 & 0.2652 & 0.2648 & 0.15 & $\mathrm{~m}$ \\
\hline 103 & 0.2926 & 0.2924 & 0.07 & w \\
\hline 222 & $\sim 0.2973$ & 0.2992 & 0.64 & $\mathrm{vw}$ \\
\hline 321 & & 0.3121 & & \\
\hline 312 & & 0.3212 & & \\
\hline 400 & 0.3294 & 0.3304 & 0.30 & $\mathrm{~m}$ \\
\hline 213 & & 0.3358 & & \\
\hline 330 & & 0.3504 & & \\
\hline 411 & & 0.3532 & & \\
\hline 004 & & 0.3740 & 0.00 & \\
\hline 303 & $0.3 / 40$ & 0.3743 & 0.08 & $\mathrm{~m}$ \\
\hline 402 & & 0.3796 & & \\
\hline 114 & & 0.3918 & & \\
\hline 332 & & 0.3972 & & \\
\hline 204 & & 0.4089 & 0.02 & yyw \\
\hline 323$\}$ & 0.4090 & 0.4091 & 0.02 & vvw \\
\hline 422 & & 0.4140 & & \\
\hline
\end{tabular}

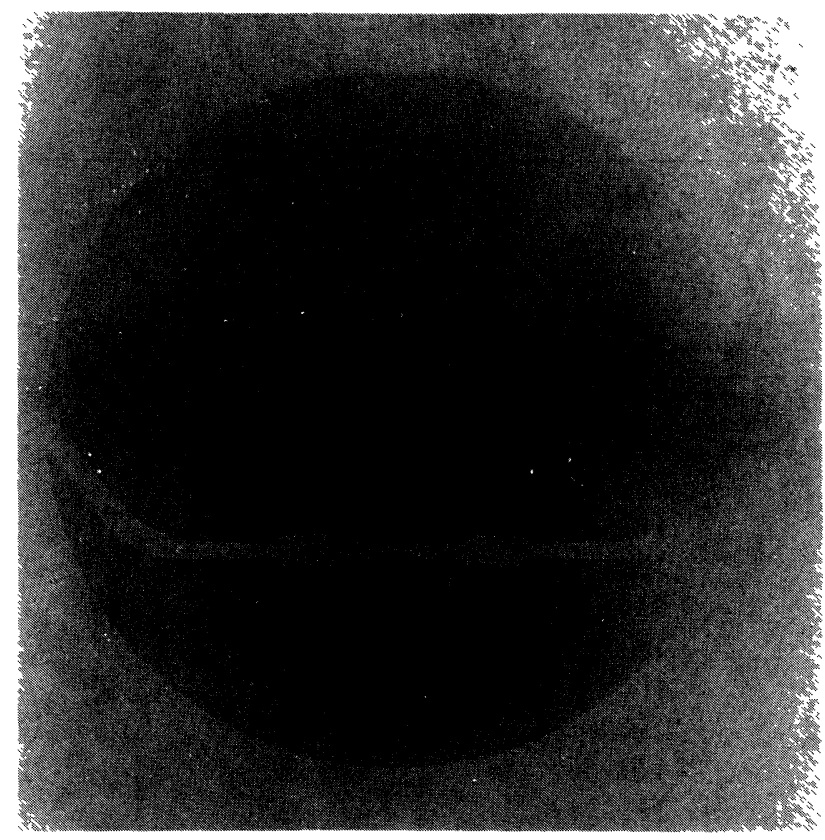

Fig. 7. - Powder diffraction pattern from a sample in the lamellar phase $\mathrm{L}_{\alpha}$ (the sample is made in the usual way at a fixed composition SDS $70.0 \% ; \mathrm{T}=55^{\circ} \mathrm{C}$; sample to film distance $517 \mathrm{~mm}$ ). The first diffraction order, which is very sharp corresponds to the repetition of the bilayers $\left(d_{\mathrm{T}}=\frac{2 \pi}{Q}=34.3 \AA\right)$. The diffuse band at small angles, corresponds to the correlations of defects, which are staggered in adjacent bilayers (their pseudo-period is $\sim 60 \AA$ ) ; it is argued that these defects are heterophase fluctuations of the tetragonal phase into the lamellar phase.

concentration boundary of the phase. Remarkably the repeat period of the bilayers $(d=34.3 \AA)$ matches the thickness of the primitive unit cell of the tetragonal phase $\mathrm{T}_{\alpha}(c / 2=33.6 \AA)$. A diffuse band of scattering is also observed at small angles. As the system is periodic in the direction normal to the bilayers, this diffuse band must correspond to correlations in directions away from the normal to the bilayers (Fig. 7). This band has been studied in detail in previous neutron scattering experiments [20] ; it is produced by defects of the bilayers, which are propagated in a staggered configuration from one bilayer to the next.

\section{Discussion.}

So far we have used the diffraction patterns to determine the shape and dimensions of the unit cell for each structure. The transformations of this unit cell through the series of mesophases are remarkable because they follow a nearly continuous path : from the two-dimensional cell of the hexagonal phase to the two-dimensional monoclinic cell (no discontinui-

ty at high temperature) and to the three-dimensional rhomboedral cell (small discontinuity, but the structures are closely related), then to the cubic phase (a special case of the previous structure) and to the tetragonal phase (a distortion of the cubic cell) and finally to the lamellar phase (discontinuous transition but with strong heterophase fluctuations).

However to describe the structures we also need to know how each unit cell is filled : what are the shapes of the amphiphilic aggregates, and what are their locations within the cell. This knowledge is also required for understanding the physical reasons for the phase changes; indeed, it is the shape and the packing of the aggregates which are directly controlled by the intermolecular forces.

Classically, this problem is dealt with in two steps. In the first step, chemical constraints pertaining to the natures of the molecules and their geometry are introduced to produce a choice of possible structures which have the correct symmetry $[1,10]$. In a second step, these structures are checked against the observed patterns by calculating the relative amplitudes of the diffraction lines which they would produce. An example of this methodology is given in the analysis of the $\mathrm{R}$ and $\mathrm{T}$ structures by Luzzati et al. 
$[5,8]$. Here we restrict ourselves to the first step of the analysis.

The main constraint commonly involved in structural analysis is the segregation of water from the hydrocarbon chains of the amphiphilic molecules. Accordingly, from the known composition of the mesophase we obtain the relative volumes of aggregates and interaggregate water ; from the volume of the unit cell we then calculate the volume of the aggregates in the cell. Also, for the same reason (no water in the aggregates), we know that at least one dimension of the aggregates must remain smaller than or equal to twice length of an extended molecule. Next, we also know from the work of Luzzati et al. [1] that, as temperature and/or water content increase, the area per polar head at the water-lipid interface never decreases, even when phase boundaries are crossed.

Accordingly, for each postulated structure, one should calculate the location of the interface and the corresponding area per polar head, and verify that this value matches those of the neighbouring phases. Finally, arguments related to the strength of interaggregate forces (repulsions between opposing surfaces) may also exclude some of the possible structure.

First consider the hexagonal phase. This structure is made of SDS cylinders whose radius remains shorter than the extended length of a SDS molecule $(17.6 \AA)$. As the concentration of SDS rises, this radius increases as well; at the boundary to the first monoclinic phase, at a SDS concentration of $57 \%$, we find that the edge of the unit cell is $47.1 \AA$, which yields a radius of $18.3 \AA$ for the cylinders. This is the largest cylinder radius which is compatible with the length of an extended SDS chain. Beyond this point, the changes in the relative volumes of SDS and water will require either that the cylinders grow to a non circular cross section, or that the thickness of the water layers decrease rapidly. The observed transition to a two-dimensional monoclinic phase shows that the first option costs less in free energypresumably because it allows the opposing surfaces to remain further from each other. Moreover, the same argument shows that the cross sections of the aggregates must be aligned alone the diagonal of the unit cell, otherwise their edges would be too close to each other.

To some extent, similar arguments can be invoked to explain the transition to the next rhomboedral phase $\left(R_{\alpha}\right)$. Indeed, at the upper end of the $\mathbf{M}_{\alpha}$ phase, the width of the aggregates would rise to $46 \AA$, for an estimated thickness of $30 \AA$; given the size of the unit cell in the $M_{\alpha}$ phase, such aggregates would nearly touch each other (edge to edge distance $\sim 5 \AA$ ). This configuration would be highly unfavorable because of the electrostatic repulsions between the polar groups of adjacent aggregates; it is much easier for the system to fuse the aggregates two by two into ribbons leaving larger spacings between them. In this way the doubling of the unit cell in one direction could be driven by interaggregate forces. Still, one has to explain why this unit cell also becomes finite along the length of the aggregates ; because interaggregate forces will not produce a modulation of the ribbons along their length, the effect of intra-aggregate forces must be considered instead. The easiest way to rationalize this modulation is to follow the classical argument which relates the curvature of the amphiphile/water interface to the relative concentrations of amphiphile and water $[15,28]$; generalize it to the mean curvature (for surfaces with heterogeneous curvature) and assume that the ribbons become connected to each other at regular intervals : in this way they would decrease the mean curvature of their surfaces by introducing a saddle splay geometry (negative Gaussian curvature). Of course other types of modulation along the length of the ribbons are possible ; however the one proposed above has the advantage of being consistent with a likely structure for the cubic phase, which is reached by a continuous deformation of this rhomboedral phase.

Next consider the cubic phase. This structure has the highest symmetry of all the three dimensional phases; moreover it can be reached through a continuous deformation from the other three dimensional structures which surround it. Therefore the determination of its structure is a key for understanding the other phases ; (it might also explain why the transformation of an array of ribbons to a stack of bilayers has to proceed through three dimensional phases).

Fortunately the spectra of the cubic phase show a large number of lines, (up to 14), which leave little uncertainty to the contents of the unit cell. We know that it is body centred, i.e. it contains one class of objects which pass through its vertices and another class which pass through its centre. Among the 6 spaces groups which are crystallographically possible, we choose the group $\operatorname{Im} 3 \mathrm{~m}$, as its structure may correspond to the third minimal surface defined by Schwarz [16] : in this case the structure is made of two interpenetrated systems of rods; the unit of each system is made of 6 rods (along the basic vectors of the unit cell) which meet at right angles at a vertex of the unit cell (for one system) or on its centre (for the other system) [29].

The existence of this structure in amphiphilic systems is important because it matches the predictions of Sadoc and Charvolin [30] which are based on the concept of bilayer frustration. A full confirmation will require the calculation of the form factors for all possible structures and their comparison with the measured intensities. 
Finally the tetragonal phase is the one which allows the stratification of the structure before it is transformed into a stack of lamellae. Because this phase can be described as a deformation of the cubic phase, it is reasonable to assume that it is based on rods running at right angles through a body centred cell. Because the period of this phase is exactly twice times the period of the lamellar phase we presume that the unit cell contains 2 planes of rods. Then the stratification of the original cubic structure is achieved by lowering the coordination number of the vertices, to 4 (in this case each plane is a square lattice of rods, and these lattices are staggered on top of each other in the unit cell). Therefore the structure appeared to be the same as that of the T phase observed previously in anhydrous long chain calcium soaps by Luzzati et al. [8].

The last transition to be considered is that from the tetragonal phase to the lamellar phase. We have seen above the way in which the tetragonal phase approaches the lamellar structure through a layering whose period matches the repetition of the bilayers in the lamellar phase. The converse process shows up on the other side of the transition ; indeed our previous neutron scattering experiments [20] show that lamellar mesophases located near this boundary are riddled with defects whose correlations resemble the diffraction pattern of the tetragonal phase. Therefore we argue that the ordering in rods or square lattices of the tetragonal phase still shows up as heterophase fluctuations in the lamellar phase.

\section{Conclusion.}

We have identified 4 intermediate mesophases between the hexagonal phase and the lamellar phase of the SDS/water system. Their structures show that the system manages to cross from a situation with homogeneously curved interfaces (cylinders) to another one with zero curvature (bilayers) in a nearly continuous way. This process occurs through the production of structures with heterogeneous curvature or with negative Gaussian curvature (saddle-splay geometry). This idea was proposed earlier by Alpérine [31] in the context of ternary systems where the segregation of two amphiphile resulted in the production of mesophases with a heterogeneous curvature (hexagonal/rectangular/lamellar phases sequence) $[6,7]$. Here we take it up again for the SDS/water system, where the production of heterogeneous curvature is expected to be more difficult since it will involve the separation of SDS molecules with and without condensed counterions.

The first step is the transformation of the cylinders into ribbons; then the ribbons become connected to each other at regular intervals to form a three dimensional array of rods. Subsequently this array is distorted to give a layered structure where the layers are spaced as in a lamellar phase but retain in the other directions the correlations of the cubic phase. The last step is the transition to a system of flat layers, which occurs partly at the transition to the lamellar phase and partly through the range of the lamellar phase.

At this stage the only physical parameter which appears to change in a consistent way through this series of structures is the mean curvature of the interfaces : this is largest for cylinders, smaller for ribbons, then presumably very small for the saddle splay structures of the cubic and tetragonal phase ; then the transition to the lamellar phase sets not only the mean curvature but also the Gaussian curvature to zero. Still a more precise determination of the shapes of the aggregates would be required to prove that the mean curvature is indeed the factor which drives the production of intermediate mesophases. These calculations are currently under way.

\section{Acknowledgments.}

It is a pleasure for us to thank J. Wagener for his help and technical advice in the design of the cells, J. P. Benoit and P. Vachette for their assistance and advice in the $\mathrm{X}$-ray scattering experiments performed at LURE. Thanks are also due to G. J. T. Tiddy for having given us the opportunity to compare our mesophases with those made with SDS purified at Unilever. Some of the ideas expressed in this paper arose from discussions with $\mathrm{J}$. Charvolin and A. M. Levelut ; we gratefully acknowledge their part in this work.

\section{References}

[1] Luzzati, V. Mustacchi, H., Skoulios, A. and Husson, F., Acta Crystallogr. 13 (1960) 660.

Husson, F., Mustacchi, H. and Luzzati, V., Acta Crystallogr. 13 (1960) 668.

[2] Luzzati, V., in Biological Membranes, D. Chapman (Ed.) (Academic Press, New York) 1968, p. 71123.

[3] Luzzati, V. and Tardieu, A., Annu. Rev. Phys. Chem. 25 (1974) 79-82.
[4] Charvolin, J. and Tardieu, A., in Solid State Phys., F. Seitz and D. Turnbull (Eds.) (Academic Press, New York) 1978, 14, p. 209.

[5] Luzzati, V., Gulik-Krzywicki, T. and TARdieu, A., Nature 218 (1968) 1031.

[6] Alperine, S., Hendrikx, Y. and Charvolin, J., J. Physique Lett. 46 (1985) L-27.

[7] Hendrikx, Y. and Charvolin, J., J. Physique 42 (1981) 1427. 
[8] Luzzati, V., Tardieu, A. and Gulik-Krzywicki, T., Nature 217 (1968) 1028.

[9] Tardieu, A., Thesis, Université Paris Sud-Orsay (1972).

[10] Luzzati, V. and Spegt, P. A., Nature 215 (1967) 701-704.

Luzzati, V., TARdieu, A., Gulik-Krzywicki, T., Rivas, E. and Reiss-Husson, F., Nature 220 (1968) 485-488.

. [11] Tardieu, A. and Luzzati, V., Biochim-Biophys. Acta 219 (1970) 11-17.

Longley, W. and Mc Intosh, T. J., Nature 303 (1983) 612-614.

[12] LuZzati, V., Mariani, P. and Gulik-KrzYwicki, Lecture presented at the workshop Physics of Amphiphilic layers Les Houches (Feb. 1987).

[13] Hendrikx, Y., Charvolin, J., Rawiso, M., Liebert, L. and Holmes, M. C., J. Phys. Chem. 87 (1983) 3991.

[14] Tiddy, G. J. T., Phys. Rep. 57 (1980) 1.

[15] Skoulios, A., Adv. Colloid Interface Sci. 1 (1967) 79.

[16] SCHWARZ, H. A., Gesammelte Mathematische Abhandlung, 1 (Springer, Berlin) 1890.

[17] Ekwall, P., in Advances in Liquid Crystals, G. H. Brown (Ed.) (Academic Press, New York) 1 (1975) 1.

[18] Charvolin, J., J. Chim. Phys. 80 (1983) nº 1.

[19] Kekicheff, P., Cabane, B. and Rawiso, M., $J$. Colloid Interface Science 102 (1984) 51.
[20] Kekicheff, P., Cabane, B. and Rawiso, M., J. Physique Lett. 45 (1984) L-813.

[21] KekichefF, P., Grabielle-Madelmont, C. and Ollivon, M., J. Colloid Interface Sci. (submitted).

[22] Lawrence, A. S. C., in Liquid Crystals 2, G. H. Brown (Ed.) (Gordon and Breach, London) 1 (1969) 1.

[23] Leigh, I. D., Mc Donald, M. P., Wood, R. M., Tiddy, G. J. T. and Trevethan, M. A., J. Chem. Soc., Faraday Trans. 177 (1981) 2867.

[24] Laughlin, R. G., Marrer, A. M., Marcott, C., MunYon, R. L., J. Micros. 139 (1985) 239.

[25] Wood, R. M. and MC Donald, M. P., J. Chem. Soc. Faraday Trans. 181 (1985) 273.

[26] Limblom, G., Larsson, K., Johansson, L., FonTEll, K. and Forsen, S., J. Am. Chem. Soc. 101 (1979) 5465.

Fontell, K., Mol. Cryst. Liq. Cryst. 63 (1981) 59.

[27] LARsSON, K., Nature 304 (1983) 664.

[28] Winsor, P. A., Chem. Rev. 68 (1968) 1.

[29] Gunning, B. E. S., Protoplasma 60 (1965) 111-130.

[30] Sadoc, J. F. and Charvolin, J., J. Physique 47 (1986) 683.

Charvolin, J. and Sadoc, J. F., J. Physique 48 (1987).

[31] Alperine, S., Thesis, Université Paris Sud-Orsay (1985). 